\title{
Leak Localization Method for Water-Distribution Networks Using a Data-Driven Model and Dempster-Shafer Reasoning
}

\author{
Adrià Soldevila, Joaquim Blesa ${ }^{\circledR}$, Tom Nørgaard Jensen, Sebastian Tornil-Sin, \\ Rosa M. Fernández-Cantí, and Vicenç Puig ${ }^{(1)}$
}

\begin{abstract}
This article presents a new data-driven method for leak localization in water-distribution networks (WDNs). The method uses the information provided by a set of pressure sensors installed in some internal network nodes in addition to flow and pressure measurements from inlet nodes. Pressure measurements are recorded under leak-free network operation, and a WDN data-driven model of the pressure at each sensed node is adjusted. The pressure estimation from this model is complemented by a Kriging spatial interpolation technique to estimate the pressure in the nodes that are not sensed, leading to a pressure reference map. Leak localization is based on the comparison of this reference pressure map with the current pressure map that is obtained by applying Kriging directly to the pressure measurements provided by sensors. The key element in this comparison is the use of the Dempster-Shafer theory for reasoning under uncertainty. The successful application of the proposed methodology to two real-data case studies is presented.
\end{abstract}

Index Terms-Data models, fault diagnosis, leak detection, water resources.

\section{INTRODUCTION}

W ATER leaks are present to some extent in all water-distribution networks (WDNs) and are estimated to account for up to $30 \%$ of the total amount of extracted

Manuscript received July 4, 2019; revised December 12, 2019; accepted February 19, 2020. Manuscript received in final form March 16, 2020. This work was supported in part by the Ministerio de Economía, Industria y Competitividad (MEICOMP) of the Spanish Government through the Project DEOCS under Grant DPI2016-76493, in part by the Catalan Agency for Management of University and Research Grants (AGAUR), in part by the European Social Fund (ESF), and in part by the Secretary of University and Research of the Department of Companies and Knowledge of the Government of Catalonia under Grant FI-DGR 2015 (2015 FI B 00591). The work of Joaquim Blesa was supported by the Serra Húnter Program. The work of Tom Nørgaard Jensen was supported by the Innovation Fund Denmark (IFD) under Grant 6155-00002B. Recommended by Associate Editor P. F. F. Odgaard. (Corresponding author: Joaquim Blesa.)

Adrià Soldevila is with FACTIC Inc., 08650 Cabrianes, Spain (e-mail: adria.cbn@gmail.com).

Joaquim Blesa, Sebastian Tornil-Sin, and Vicenç Puig are with the Research Center for Supervision, Safety and Automatic Control (CS2AC), 08022 Terrassa, Spain, and also with the Institut de Robòtica i Informàtica Industrial (CSIC-UPC). 08028 Barcelona, Spain (e-mail: joaquim.blesa@upc.edu; sebastian.tornil@upc.edu; vicenc.puig@upc.edu).

Tom Nørgaard Jensen is with the New Product Development \& Control Systems, Alfa Laval Aalborg A/S, 9100 Aalborg, Denmark (e-mail: noergaard_jensen@ hotmail.com).

Rosa M. Fernández-Cantí is with the Research Center for Supervision, Safety and Automatic Control (CS2AC), 08022 Terrassa, Spain (e-mail: rosa.mari.fernandez@upc.edu).

Color versions of one or more of the figures in this article are available online at http://ieeexplore.ieee.org. water [1]. This is a very significant amount since water is a precious resource, especially in many parts of the world where it is necessary to satisfy the water demands of a growing population and sometimes in the presence of drought periods that are increased by climate change. Fault diagnosis and security in water systems are key challenges that will become even more crucial in the years ahead [2].

Several works have been published dealing with the leak detection and isolation (localization) problem in WDNs (see [3] and references therein). For example, in [1], a review of transient-based leak detection methods is offered. In [4], a method is proposed to identify leaks using blind spots based on previous leak detection that uses the analysis of acoustic and vibration signals [5] and models of buried pipelines to predict wave velocities [6]. More recently, [7] presents a method to localize leaks using support vector machines (SVMs) that analyze the data obtained by a set of pressure control sensors installed in a pipeline network to localize and calculate the size of the leak. The use of $k$-nearest neighbors and the Bayesian and neurofuzzy classifiers in leak localization have been also recently proposed in [8] and [9], respectively.

Some of the recently proposed leak localization methods use pressure sensors inside the WDNs. Pressure sensors are cheaper and easier to install than flow sensors. Therefore, a setup with a few pressure sensors installed in the WDN is an attractive option for utilities. One such setup is presented in [11], where a model-based method that relies on the pressure measurements and a leak sensitivity analysis is proposed. In this methodology, pressure residuals, i.e., differences between pressure measurements provided by sensors and the corresponding estimations obtained by using a hydraulic model, are used. These residuals are computed online and compared against associated thresholds that take into account the effects of modeling uncertainty and noise. When some of the residuals exceed their thresholds, the residuals are matched against the leak sensitivity matrix in order to discover which of the possible leaks is present. Although this approach has good efficiency under ideal conditions, its performance decreases due to the nodal demand uncertainty and measurement noises [12], [13]. The methodology has been improved in [14], where a comparison of several geometric approaches for leak localization is compared and analysis along a time horizon is proposed. Additional improvements are achieved by processing the residuals using classifiers [8], [9]. 
In this article, we propose a new method to deal with the leak localization problem in WDNs or in partitioned zones called district metered areas (DMAs), where the flow and pressure at the inlets are measured and some pressure sensors are placed inside. It is, here, assumed that the leak detection task is already been performed, e.g., using some of the approaches reviewed in [1]. The proposed method is based on three key ideas:

1) use of a data-driven adjusted model to estimate nominal (without a leak) expected pressure values according to the current operating conditions in the internal nodes that are equipped with pressure sensors;

2) use of the Kriging spatial interpolation technique to estimate the pressure at the network nodes that are not equipped with sensors based on the hydraulic proximity;

3) use of the Dempster-Shafer (DS) theory for reasoning under uncertainty to analyze the differences between the nominal and the currently observed network behavior.

The proposed method has two clear benefits. First, it is a data-driven method that does not require the computation, the recursive updating, and the difficult calibration of a hydraulic model. An estimation of the hydraulic resistance between nodes in the network is used instead. Second, it only needs historical data from normal operation behavior in contrast to conventional data-driven methods that require historical data for all the considered abnormal behaviors.

The rest of this article is organized as follows. In Section II, the data-driven model of the network and the spatial interpolation based on Kriging are presented, and the leak localization problem is introduced as the evaluation of residuals generated by the data-driven model. Section III proposes an improved localization using DS time reasoning. The method is summarized in Section IV. Section V illustrates the application of the leak localization methodology to two real DMAs. Finally, Section VI draws the main conclusions of this work.

Notations: Matrices are denoted using capital and bold letters, while lower and bold letters denote vectors, and italic letters denote scalars. Sets and graphs are represented using calligraphic letters.

\section{WDN DATA-DRIVEN MODEL}

A WDN can be described by a directed graph $\mathcal{G}=\{\mathcal{V}, \mathcal{E}\}$. Here, $\mathcal{V}=\left\{v_{1}, \ldots, v_{n_{m}}\right\}$ is the set of vertices that represent connections between the components of the network, i.e., the $n_{n}$ internal nodes and the $n_{r}$ inlet nodes, giving a total number of nodes $n_{m}=n_{n}+n_{r}$. The set $\mathcal{E} \in \mathcal{V} \times \mathcal{V}$ with $\mathcal{E}=\left\{e_{1}, \ldots, e_{n_{p}}\right\}$ is the set of edges, which represents the $n_{p}$ pipes of the network. A path $\mathcal{P}$ in the graph $\mathcal{G}$ is a sequence $\left\{x_{i}\right\}_{i=1}^{\ell}$ such that $x_{i} \in \mathcal{V}, x_{i} x_{i+1} \in \mathcal{E}$, and $x_{i} \neq x_{j}$ for every pair $i, j \in\{1,2, \ldots, \ell\}$. Furthermore, we consider that the edge $e_{j} \in \mathcal{P}$ if $e_{j}=x_{k} x_{k+1}$ with $x_{k} \in \mathcal{P}$ and $x_{k+1} \in \mathcal{P}$.

Let $\mathbf{p}$ be the vector of absolute pressures at the nodes and $\Delta \mathbf{p}$ be the vector of differential pressures across the pipes, both in meters water column [mwc], and then

$$
\Delta \mathbf{p}=\mathbf{H}^{T} \mathbf{p}=f(\mathbf{q})-\mathbf{H}^{T} \mathbf{h}
$$

where $\mathbf{p} \in \mathbb{R}^{n_{m}}$ and $\mathbf{q} \in \mathbb{R}^{n_{p}}$ are the vectors of volumetric flow in the edges in cubic meters per second $\left[\mathrm{m}^{3} / \mathrm{s}\right], \mathbf{H}$ with size $n_{m} \times n_{p}$ is the incidence matrix, ${ }^{1} f: \mathbb{R}^{n_{p}} \rightarrow \mathbb{R}^{n_{p}}$, and $f(\mathbf{q})=$ $\left(f_{1}\left(q_{1}\right), \ldots, f_{n_{p}}\left(q_{n_{p}}\right)\right)$. The function $f_{i}\left(q_{i}\right)$ describes the flow dependent pressure drop due to the hydraulic resistance in the $i$ th edge.

For turbulent flow in the pipes, the Darcy-Weisbach equation is a good approximation of the pressure drop due to the hydraulic resistance of the pipe [16]. The expression in the $i$ th pipe is given by

$$
f_{i}\left(q_{i}\right)=\frac{8 C_{i} L_{i}}{\pi^{2} g D_{i}^{5}}\left|q_{i}\right| q_{i}
$$

where $C_{i}$ is the coefficient of surface resistance (which is, in part, determined by the flow, but we will here consider it constant) with adimensional units, $L_{i}$ is the length of the pipe in $[\mathrm{m}], g$ is the gravitational acceleration in $\left[\mathrm{m} / \mathrm{s}^{2}\right]$, and $D_{i}$ is the diameter of the pipe in [m].

The term $\mathbf{H}^{T} \mathbf{h}$ is the pressure drop across the pipes due to the difference in geodesic level (i.e., elevation), in meters [m], between the ends of the pipes, with $\mathbf{h} \in \mathbb{R}^{n_{m}}$ the vector of geodesic levels at each vertex.

Knowledge of the length and diameter of all the pipes in the network as well as their interconnections (topological structure) and the geodesic level at all nodes is required. Therefore, we assume that these values can be obtained from an available database.

\section{A. Interpolation Between Boundary Conditions}

Here, we will present the reduced-order model that will be used for calculating the expected nominal pressures at the measured internal nodes, based on the known values of flows and pressures at the inlets and the previously measured values in the sensed nodes. The derivation of the model is presented in [17].

Nodes in a network can be classified as inlet nodes (directly connected to tanks or reservoirs) and inner nodes (associated with network internal junctions and consumer nodes). In the following, variables associated with inlet nodes are indicated with a (1) superscript, while variables associated with inner nodes present a (2) superscript. For each node (inlet or inner node), the three variables of interest are the elevation, the pressure, and the head, represented in this article with the letters "h," "p," and "y," respectively. For each node $i$, the head is defined as the addition of the elevation and the pressure at the node, i.e., $y_{i}=h_{i}+p_{i}$.

Now, as suggested in [18], consider the case where

$$
\mathbf{y}^{(1)}=\mathbf{p}^{(1)}+\mathbf{h}^{(1)}=\kappa \mathbb{1}
$$

for some $\kappa \in \mathbb{R}$ that is the total head at the inlets in [mwc], and $\mathbb{1}$ denotes the vector consisting of ones.

The constraint (3) has the physical implication that the total head at all inlets must be equal at all times. At first glance, this seems like a hypothetical assumption. However, it appears that at least in some networks, this assumption is fulfilled, which we have observed through measurements of the inlet pressures (as, e.g., the real networks considered as the case study in this

\footnotetext{
${ }^{1}$ The incidence matrix of the graph, which describes the interconnection of vertices and edges, is defined as usual in graph theory (see [15]).
} 
article). Moreover, we can refer the reader to [17], in which a discussion is found as to why controllers would have to fulfill this assumption at least in networks with the low total consumption. For networks fulfilling (2) and (3), we have the following proposition from [17].

Proposition 1: If the vector $\mathbf{p}^{(1)}$ of pressures at inlet nodes fulfills (3) and $f_{i}(\cdot)$ fulfills (2), then the pressure at the $i$ th internal node follows the expression:

$$
p_{i}^{(2)}=\alpha_{i} \sigma^{2}+\kappa+\gamma_{i}
$$

where $\alpha_{i}$ and $\gamma_{i}$ are parameters dependent on the network topology and the distribution of demands in the network, and $\sigma>0$ is the total inlet flow (sum of all inlet flows) in $\left[\mathrm{m}^{3} / \mathrm{s}\right]$. The total inlet flow $\sigma$ is typically well known since inlet flows are measured.

In general, the parameter $\alpha_{i}$ in (4) is time varying. However, if the proportion of the total consumption of any particular end user at any point in time is constant, then $\alpha_{i}$ is also constant. The latter is the case if all end users follow the same profile; for instance, if all users are residential.

Since the model (4) of $p_{i}^{(2)}$ is linear in the parameters $\alpha_{i}$ and $\gamma_{i}$, standard parameter identification methods [19] can be used to identify these parameters based on measurements of $p_{i}^{(2)}, \sigma$, and $\mathbf{p}^{(1)}$ over a number of time periods. Subsequently, having identified the parameters, the model (4) can be used to predict the expected pressure $\tilde{p}_{i}^{(2)}$ at a measurement vertex since the variables $\sigma$ and $\mathbf{p}^{(1)}$ are measured and $\mathbf{h}^{(1)}$ is known.

\section{B. Pressure Estimation in Unsensed Nodes}

In practice, in real WDNs, pressure measurements $p_{i}^{(2)}$ are only available in a limited number of internal nodes due to the costs of installing and maintaining pressure sensors. The location of the installed sensors is given by the index set $\mathcal{S}$ of internal nodes equipped with sensors

$$
\mathcal{S}=\left\{s_{1}, \ldots, s_{n_{s}}\right\}
$$

where $n_{s}$ is the number of pressure sensors installed.

To obtain the pressure in the nodes without pressure sensors installed, an interpolation technique can be used. In this work, the Kriging approach is used. Kriging is a well-known interpolation method in the area of geostatistics, and it can be seen as a multivariate regression approach (see [20] for a recent review). The basic idea of Kriging is to estimate the value of a function at a given point by computing a weighted average of the known values of the function in the neighborhood of the point.

Consider that the WDN is working under some operating conditions $\mathbf{c}$ given by the total inlet flow and user demands, as proposed in [21]. Since the user demands are rarely measured, the vector c contains the total head at the inlets $\kappa$, obtained by taking into account PRVs or gravity and the total inlet flow $\sigma$.

The vector $\mathbf{q}$ consisting of flows in all the pipes in the network can be partitioned as

$$
\mathbf{q}=\mathbf{a} \sigma
$$

where a determines the distribution of flow in pipes and is unknown, and the total inlet flow $\sigma$ is typically well known since inlet flows are measured values.

It was shown in [17] (or in [22] for the single inlet case) that when the distribution of the total inlet flow among the end users remains constant (i.e., all consumers have the same consumption profile), then the vector $\mathbf{a}$ is constant as well.

Having knowledge of the absolute pressure, say $p_{i}^{(2)}$, i.e., the upstream of the $k$ th pipe, the head $y_{j}^{(2)}$ downstream the pipe can be obtained from (1) as

$$
y_{j}^{(2)}=p_{j}^{(2)}+h_{j}^{(2)}=p_{i}^{(2)}+h_{i}^{(2)}-f_{k}\left(q_{k}\right) .
$$

Now, we rewrite (2) using the definition in (6) and obtain

$$
f_{k}\left(q_{k}\right)=\frac{8 C_{k} L_{k}}{\pi^{2} g D_{k}^{5}}\left|a_{k} \sigma\right| a_{k} \sigma=\frac{8 C_{k} L_{k}}{\pi^{2} g D_{k}^{5}}\left(\left|a_{k}\right| a_{k}\right) \sigma^{2}
$$

where the latter equality is due to the fact that $\sigma>0$.

If we assume that we have knowledge of the length and diameter of pipes, we can rearrange (8) into known and unknown quantities to obtain

$$
f_{k}\left(q_{k}\right)=\overbrace{C_{k}\left(\left|a_{k}\right| a_{k}\right)}^{\theta_{k}} \frac{8 L_{k}}{\pi^{2} g D_{k}^{5}} \sigma^{2}=\theta_{k} \frac{8 L_{k}}{\pi^{2} g D_{k}^{5}} \sigma^{2} .
$$

$\theta_{k}$ is now an unknown parameter of the $k$ th pipe, and it will be estimated using the Kriging approach. Now, (7) can be expressed as

$$
\hat{y}_{j}^{(2)}=p_{i}^{(2)}+h_{i}^{(2)}-\hat{\theta}_{k} \frac{8 L_{k}}{\pi^{2} g D_{k}^{5}} \sigma^{2}
$$

where the term $\left(8 L_{k} /\left(\pi^{2} g D_{k}^{5}\right)\right) \sigma^{2}$ will be used as the independent variable in the interpolation.

Since multiple pipe paths may connect $j$ th and $i$ th vertices, we suggest to use the shortest "weighted" pipe length (e.g., weighted by pipe diameters) as the interpolation variable in the Kriging interpolation. That is, if we let $\mathcal{P}_{i j}=$ $\left\{\mathcal{P}_{i j}(1), \ldots, \mathcal{P}_{i j}(n)\right\}$ denote the set of paths connecting nodes $i$ and $j$, then we define the shortest weighted pipe length $\overline{\mathcal{D}}_{i j}$ as

$$
\overline{\mathcal{D}}_{i j}=\underset{\mathcal{P}_{i j}(k) \in \mathcal{P}_{i j}}{\arg \min } \sum_{e_{z} \in \mathcal{P}_{i j}(k)} \frac{L_{z}}{D_{z}^{5}}
$$

In other words, for every path between the two nodes, we calculate the sum of $\left(L_{z} /\left(D_{z}^{5}\right)\right)$ for all edges $e_{z}$ in the path. The path that returns the smallest sum is the one used in the interpolation.

Thus, the estimation of an unmeasured head $\hat{y}_{i}^{(2)}$ in the $i$ th node can be computed by means of a fit Kriging model [23] as

$$
\hat{y}_{i}^{(2)}(\mathbf{c}, \mathcal{S})=\mu(\mathbf{c})+\varepsilon\left(\chi(\mathbf{c}), \boldsymbol{\phi}(\mathbf{c}), \overline{\mathcal{D}}_{i:}(\mathcal{S})\right)
$$

where $\mathbf{c}$ is the vector that defines the operation conditions (i.e., total head in inlets $\kappa$ and total inflow $\sigma$ ), $\mathcal{S}$ is the sensor set defined in (5), and $\mu(\mathbf{c})$ provides a value that represents the constant part of the interpolation given a particular operating condition c. On the other hand, the function $\varepsilon\left(\chi(\mathbf{c}), \boldsymbol{\phi}(\mathbf{c}), \overline{\mathcal{D}}_{i:}(\mathcal{S})\right)$ is the spatially correlated part of the variation, where $\chi(\mathbf{c})$ is a polynomial function, $\phi(\mathbf{c})$ is the 
correlation function, and $\overline{\mathcal{D}_{i:}}(\mathcal{S})$ is a vector containing the shortest weighted pipe distances, defined in (11), between the node $i$ and the $n_{s}$ sensors, as $\overline{\mathcal{D}_{i:}}(\mathcal{S})=\left(\overline{\mathcal{D}}_{i s_{1}}, \ldots, \overline{\mathcal{D}}_{i s_{n_{s}}}\right)$. Both terms $\mu(\mathbf{c})$ and $\varepsilon(\cdot)$ are obtained in the fitting process as well as the functions $\chi(\mathbf{c})$ and $\boldsymbol{\phi}(\mathbf{c})$. The fitting process consists of a least-squares error minimization problem considering available pressure measurements and distances between the $n_{n}$ nodes and the $n_{s}$ sensors installed. The reader is referred to [21] for more details about interpolation model (12).

Finally, the pressure in the unmeasured node $i$ can be obtained from (12) by subtracting the geodesic level as

$$
\hat{p}_{i}^{(2)}(\mathbf{c}, \mathcal{S})=\hat{y}_{i}^{(2)}(\mathbf{c}, \mathcal{S})-h_{i}^{(2)} .
$$

\section{Basic Residual Computation and Evaluation}

The leak localization problem, posed as a fault detection and isolation (FDI) problem in the literature, typically assumes that only one leak can occur at a time. In addition, it is also usually assumed that leaks can only occur in the nodes of the network (e.g., as considered in [9], [11], or [24]), which makes the number of potential leaks equal to the number of nodes of the considered network.

As stated in the introduction, the proposed leak localization technique is applied after the detection of a leak in the monitored network. Therefore, the used measurements are assumed to be captured under a leaky situation. Consider the presence of a leak $l_{j}$ with magnitude $l$ and acting on the node $j$ of the network. If leak-free historical data of pressure sensors installed in inner nodes are available for all possible operating conditions, including the current operating conditions $\mathbf{c}$, then residual pressures in internal nodes can be computed as

$$
r_{i}=\tilde{p}_{i}^{(2)}(\mathbf{c})-p_{i}^{(2)}\left(\mathbf{c}^{l_{j}}\right) \quad \forall i \in \mathcal{S}
$$

where $p_{i}^{(2)}\left(\mathbf{c}^{l_{j}}\right)$ is the pressure value measured by the inner pressure sensor $i$ under boundary conditions $\mathbf{c}^{l_{j}}$ (total head and inflow in inlets) under the presence of leak $l_{j}$. On the other hand, $\tilde{p}_{i}^{(2)}(\mathbf{c})$ is its estimation considering boundary conditions $\mathbf{c}$ in a leak-free scenario that can be computed reformulating (4) as

$$
\tilde{p}_{i}^{(2)}(\mathbf{c})=\hat{\alpha}_{i} \sigma^{2}+\kappa+\hat{\gamma}_{i}
$$

where $\hat{\alpha}_{i}$ and $\hat{\gamma}_{i}$ are estimated from historical leak-free data applying classical identification methods based on least-squares estimation.

Considering the Kriging spatial interpolation to estimate the pressure in the unmeasured nodes (13) both in nonleak $p_{i}^{(2)}\left(\mathbf{c}^{\mathbf{l}_{j}}\right)$ and leak $\tilde{p}_{i}^{(2)}(\mathbf{c})$ conditions $\forall i \in \mathcal{S}$, the residual vector for all internal nodes can be approximated by

$$
\hat{\mathbf{r}}=\hat{\mathbf{p}}^{(2)}(\mathbf{c}, \mathcal{S})-\hat{\mathbf{p}}^{(2)}\left(\mathbf{c}^{l_{j}}, \mathcal{S}\right)
$$

Here, the following holds.

1) $\hat{\mathbf{p}}^{(2)}(\mathbf{c}, \mathcal{S})=\left(\hat{p}_{1}^{(2)}(\mathbf{c}, \mathcal{S}), \ldots, \hat{p}_{n_{n}}^{(2)}(\mathbf{c}, \mathcal{S})\right)$ is the vector that approximates the pressure map in the WDN under operating conditions $\mathbf{c}$ in a leak-free scenario (reference map).

2) $\hat{\mathbf{p}}^{(2)}\left(\mathbf{c}^{l_{j}}, \mathcal{S}\right)=\left(\hat{p}_{1}^{(2)}\left(\mathbf{c}^{l_{j}}, \mathcal{S}\right), \ldots, \hat{p}_{n_{n}}^{(2)}\left(\mathbf{c}^{l_{j}}, \mathcal{S}\right)\right)$ is the vector that approximates the pressure map in the WDN under operating conditions $\mathbf{c}^{l_{j}}$ in a leak scenario with a magnitude $l$ in node $j$.

Then, leaky node localization can be estimated as the one with the largest pressure residual component (as used in [25] and [26] and analytically justified in [27]), that is

$$
\hat{J}=\underset{i \in\left\{1, \ldots, n_{n}\right\}}{\arg \max }\left\{\hat{r}_{i}\right\}
$$

where $\hat{r}_{i} \forall i \in\left\{1, \ldots, n_{n}\right\}$ are the components of residual vector (16).

The performance when using the leak localization defined in (17) will depend on the number of sensors, their location in the WDN, and the accuracy of the data-driven model (4) to predict the pressure under the operational conditions $\mathbf{c}$ and leak-free conditions.

Note that according to (10), the consumption $(\sigma)$ affects the value of the residual, providing larger residuals as the consumption increases for the same leak size. To deal with that issue and to obtain a robust leak localization, the DS reasoning is used here to integrate the temporal development of the residual (16).

\section{Dempster-Shafer ReAsoning}

\section{A. Dempster-Shafer Evidence Theory}

The DS evidence theory [28], [29] is a general framework for reasoning with the uncertainty that allows to fuse information from different sources. In the same way, evidence from the same source but at different time instants can also be considered. In [30], the use of the DS reasoning approach is proposed as an alternative to the Bayes reasoning when ambiguous hypotheses exist. This is the case of the leak localization approach with a reduced number of sensors since several leaks present a similar pressure pattern. On the other hand, the Bayes reasoning considers all of the supported hypotheses must be mutually exclusive. This is the main motivation for using DS reasoning in this article.

Given a set of individual hypothesis $\mathcal{A}_{i}$, the DS theory considers all the possible combinations, i.e., the power set $\mathcal{P}$. For each subset $\mathcal{A}$ of the power set $\mathcal{P}$, three quantities are defined: the mass $m(\mathcal{A})$, the belief $\operatorname{bel}(\mathcal{A})$, and the plausibility $p l(\mathcal{A})$. The mass is associated with the information provided by a given source, and it can also be considered as a subjective probability. Hence, a probability mass function (pmf) is a function that satisfies: $m(\emptyset)=0 ; m(\mathcal{A}) \in[0,1] \forall \mathcal{A} \in \mathcal{P}$; and $\sum_{\mathcal{A} \in \mathcal{P}} m(\mathcal{A})=1$. The belief $\operatorname{bel}(\mathcal{A})$ is defined as the sum of all the masses of all the subsets of $\mathcal{A}$, i.e., $\operatorname{bel}(\mathcal{A})=$ $\sum_{\mathcal{B} \mid \mathcal{B} \in \mathcal{A}} m(\mathcal{B})$. The plausibility $\operatorname{pl}(\mathcal{A})$ is the sum of all the masses of the subsets of $\mathcal{P}$ that intersects $\mathcal{A}$, i.e., $\operatorname{pl}(\mathcal{A})=$ $\sum_{\mathcal{B} \mid \mathcal{B} \cap \mathcal{A} \neq \emptyset} m(\mathcal{B})$. The belief and the plausibility define an interval where the true probability of the considered hypothesis lies, i.e., $\operatorname{bel}(\mathcal{A}) \leq m(\mathcal{A}) \leq \operatorname{pl}(\mathcal{A})$.

When there are two sources of information, Dempster's rule of combination can be used. Being $m_{g 1}$ the mass assignment associated with the first source and $m_{g 2}$ the one associated 
with the other, the joint mass is computed as

$$
\begin{aligned}
m_{g 1, g_{2}}(\mathcal{A}) & =\left(m_{g_{1}} \oplus m_{g_{2} 2}\right)(\mathcal{A}) \\
& =\frac{\sum_{\mathcal{B} \cap C=\mathcal{A} \neq \emptyset} m_{g_{1}}(\mathcal{B}) m_{g_{2}}(\mathcal{C})}{1-\sum_{\mathcal{B} \cap \mathcal{C}=\emptyset} m_{g_{1}}(\mathcal{B}) m_{g_{2}}(\mathcal{C})}
\end{aligned}
$$

where $\mathcal{B}$ and $\mathcal{C}$ are subsets of $\mathcal{P}$ and $\oplus$ is the direct sum [29]. The constraint $\mathcal{B} \cap \mathcal{C}=\mathcal{A} \neq \varnothing$ limits the interaction of the probability mass functions only to the shared hypotheses, and the constraint $\mathcal{B} \cap \mathcal{C}=\emptyset$ contains the rest of combinations of probability mass functions. More precisely, the denominator in (18) is for normalizing the result, i.e., the sum of the resulting mass probabilities of the resulting set of common hypotheses $\mathcal{A}(m(\mathcal{A}))$ is one. This Dempster's rule of combination considering three different sources of information was used in [31] to perform the leak localization task.

Dempster's rule of combination (18) can be formulated for time reasoning [32] as

$$
m_{t_{1}, t_{2}}(\mathcal{A})=m_{t_{1}}(\mathcal{A}) \oplus m_{t_{2}}(\mathcal{A})
$$

where $t_{1}$ and $t_{2}$ are two different time instants for the same set of hypotheses $\mathcal{A}$. More precisely, for a particular hypothesis $\mathcal{A}_{i}$ in the set $\mathcal{A}$, we can say

$$
\begin{aligned}
& m_{t_{1}, t_{2}}\left(\mathcal{A}_{i}\right) \\
& =\frac{1}{\Lambda} \times \sum_{j=1, \ldots, A \mid \mathcal{A}_{i} \subseteq \mathcal{A}_{j}}\left(m_{t_{1}}\left(\mathcal{A}_{i}\right) m_{t_{2}}\left(\mathcal{A}_{j}\right)+m_{t_{1}}\left(\mathcal{A}_{j}\right) m_{t_{2}}\left(\mathcal{A}_{i}\right)\right)
\end{aligned}
$$

where $A$ is the number of hypotheses contained in the set $\mathcal{A}$ and $\Lambda$ is a normalization factor such that $\sum_{i=1}^{A} m_{t_{1}, t_{2}}\left(\mathcal{A}_{i}\right)=1$.

\section{B. Probability Mass Functions for Leak Localization}

The presented DS framework can be applied to the leak localization problem. Leaks in network nodes can be considered as the individual hypothesis $\mathcal{H}_{i}$, and a probability mass function can be associated with normalized pressure residual components of vector (16) as

$$
m_{t}\left(\mathcal{H}_{i}\right)=\frac{\hat{r}_{i}(t)-\min (\hat{\mathbf{r}}(t))}{\sum_{j=1}^{n_{n}}\left(\hat{r}_{j}(t)-\min (\hat{\mathbf{r}}(t))\right)}
$$

where the size of the hypothesis set $\mathcal{H}$ is the number of internal nodes of the network $n_{n}$ and

$$
\sum_{i=1}^{n_{n}} m_{t}\left(\mathcal{H}_{i}\right)=1
$$

In order to illustrate the proposed leak localization method, we will consider the simplified WDN of Hanoi (Vietnam) introduced in [33] and depicted in Fig. 1. This network has one reservoir, 31 internal nodes $\left(n_{n}=31\right)$, and 34 pipes $\left(n_{p}=34\right)$, and it is assumed that three pressure sensors are placed in internal nodes with indices 2,8 , and $24\left(n_{s}=3\right)$. Thus, for this particular network, the hypothesis set $\mathcal{H}$ will be $n_{n}=31$, one for each potential leak location considered, i.e., inner node.

However, the use of these probability mass functions presents the particularities that the complete set of hypotheses is composed of singletons (i.e., inside each hypothesis, there is no uncertainty among single hypothesis) and fulfills the

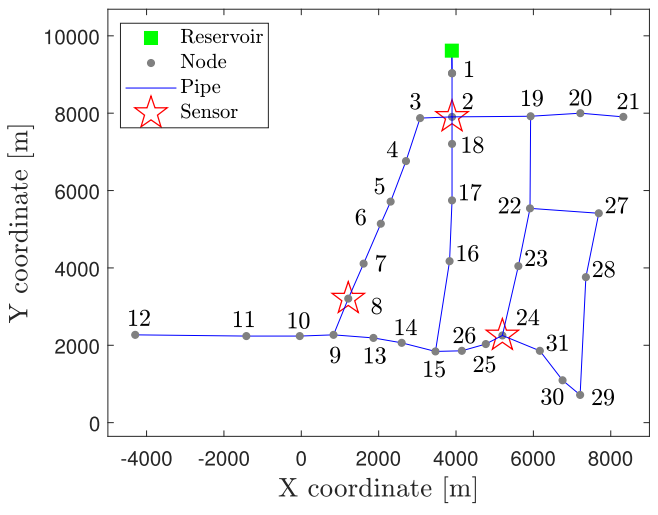

Fig. 1. Simplified Hanoi WDN.

condition (22). Thus, the application of Dempster's rule of combination is analogous to the application of the Bayes rule [34]. Thus, the potential of Dempster's rule of the combination will not be fully exploited. Thus, with that aim, additional hypotheses sets of clustered singleton hypotheses from the set $\mathcal{H}$ are proposed by taking into account spatial and hydraulic information.

\section{Clustering}

According to section II-B, as nearby nodes present similar hydraulic behavior [35], the sensor relation to the nodes of the network is analyzed using the hydraulic relation given by the shortest weighted pipe distance $\overline{\mathcal{D}}$. This weighted pipe distance can also be used to infer a set of node clusters that can be considered as an additional hypothesis under the DS framework.

First, $n_{s}$ clusters are created, each one of them associated with one of the network nodes that are equipped with pressure sensors. Each cluster around a sensed node includes all the network nodes that present a minimum hydraulic distance to this particular sensed node. The obtained set of clusters defines a partition of the network similar to a Voronoi diagram. Formally, the cluster associated with a given sensed node $l$ is defined as

$$
\mathcal{K}_{l}^{(\mathcal{L})}=\left\{v_{i}^{(2)} \in \mathcal{V}^{(2)} \mid \underset{j \in \mathcal{S}}{\arg \min }\left\{\overline{\mathcal{D}}_{i j}\right\}=l\right\}
$$

where $l \in\left\{1, \ldots, n_{s}\right\}$. The whole set of clusters (one for each sensed node) can be represented as $\mathcal{K}^{(\mathcal{L})}$.

In the simplified WDN of Hanoi, where three pressure sensors are considered, the computed clusters associated with each sensed node are depicted in Fig. 2.

Furthermore, some sensors can be close in hydraulic terms, and their measurements can be significantly affected by a leak in the nodes clustered in another cluster from a nearby sensor. To take that into account, a second clustering is performed.

These additional clusters are based on the previous clusters $\mathcal{K}^{(\mathcal{L})}$ and are computed by implementing three steps inside a loop. The loop considers each possible pair $\mathcal{K}_{i}^{(\mathcal{L})}, \mathcal{K}_{j}^{(\mathcal{L})} \in$ $\mathcal{K}^{(\mathcal{L})}$, and at each loop iteration, the following three steps are implemented. 


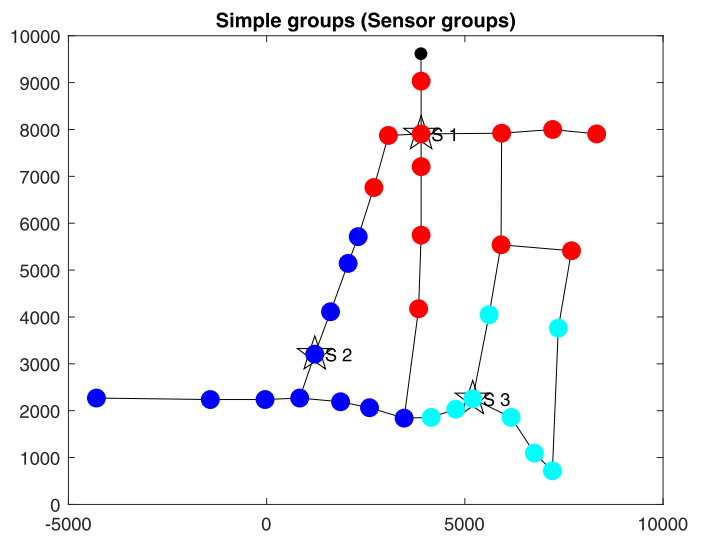

Fig. 2. $\mathcal{K}^{(\mathcal{L})}$ clusters in the simplified WDN of Hanoi.

First, for every cluster $\mathcal{K}_{j}^{(\mathcal{L})}$, the mean of the shortest weighted pipe distance between the nodes and the sensor is computed as

$$
d_{j}^{(\mathcal{L})}=\frac{1}{\left|\mathcal{K}_{j}^{(\mathcal{L})}\right|} \sum_{v_{i}^{(2)} \in \mathcal{K}_{j}^{(\mathcal{L})}} \overline{\mathcal{D}}_{i j}
$$

where $\left|\mathcal{K}_{j}^{(\mathcal{L})}\right|$ is the number of elements of the cluster $\mathcal{K}_{j}^{(\mathcal{L})}$.

Second, for every couple of clusters $\mathcal{K}_{i}^{(\mathcal{L})}$ and $\mathcal{K}_{j}^{(\mathcal{L})}$, with $i \neq j$, a threshold is set as the minimum of the mean of the shortest weighted pipe distance between the couple of sensors as

$$
\tau_{i j}=\min \left\{d_{i}^{(\mathcal{L})}, d_{j}^{(\mathcal{L})}\right\} \forall i \neq j \in \mathcal{S} .
$$

Third, the elements of a new cluster $\mathcal{K}_{k}^{(\mathcal{W})}$ can be found from the elements of the couple of clusters $\mathcal{K}_{i}^{(\mathcal{L})}$ and $\mathcal{K}_{j}^{(\mathcal{L})}$ as

$$
\mathcal{K}_{k}^{(\mathcal{W})}=\mathcal{K}_{k_{i}}^{(\mathcal{W})} \cup \mathcal{K}_{k_{j}}^{(\mathcal{W})}
$$

where

$$
\mathcal{K}_{k_{i}}^{(\mathcal{W})}=\left\{v_{l}^{(2)} \in \mathcal{K}_{i}^{(\mathcal{L})} \mid \overline{\mathcal{D}}_{l j} \leqslant \tau_{i j}\right\}
$$

and

$$
\mathcal{K}_{k_{j}}^{(\mathcal{W})}=\left\{v_{l}^{(2)} \in \mathcal{K}_{j}^{(\mathcal{L})} \mid \overline{\mathcal{D}}_{l i} \leqslant \tau_{i j}\right\} .
$$

The potential number of the new resulting clusters is $\left(n_{s} ! /\left(2 !\left(n_{s}-2\right) !\right)\right)$, but only some combinations of clusters $\mathcal{K}_{i}^{(\mathcal{L})}$ and $\mathcal{K}_{j}^{(\mathcal{L})}$ can lead to a nonempty new clusters $\mathcal{K}_{k}^{(\mathcal{W})} \neq$ $\emptyset$ in (26). Then, the real number of new clusters $w \leq$ $\left(n_{s} ! /\left(2 !\left(n_{s}-2\right) !\right)\right)$ will depend on the sensor configuration.

All the singleton clusters obtained, if any, are not considered since they are already contained in the set $\mathcal{H}$.

Fig. 3 depicts the elements of cluster $\mathcal{K}_{1}^{(\mathcal{W})}$ that is one of the three possible additional clusters in the simplified WDN of Hanoi. This cluster is computed by means of (26) considering clusters $\mathcal{K}_{1}^{(\mathcal{L})}$ and $\mathcal{K}_{2}^{(\mathcal{L})}$ associated with sensed nodes 2 and 8 , respectively.

The first clusters $\mathcal{K}^{(\mathcal{L})}$ are converted to a new set of composed hypothesis $\mathcal{L}$ where each cluster is a composed hypothesis from the hypotheses in $\mathcal{H}$. In the same way, each cluster $\mathcal{K}^{(\mathcal{W})}$ will be a composed hypothesis from the new set of hypotheses $\mathcal{W}$.

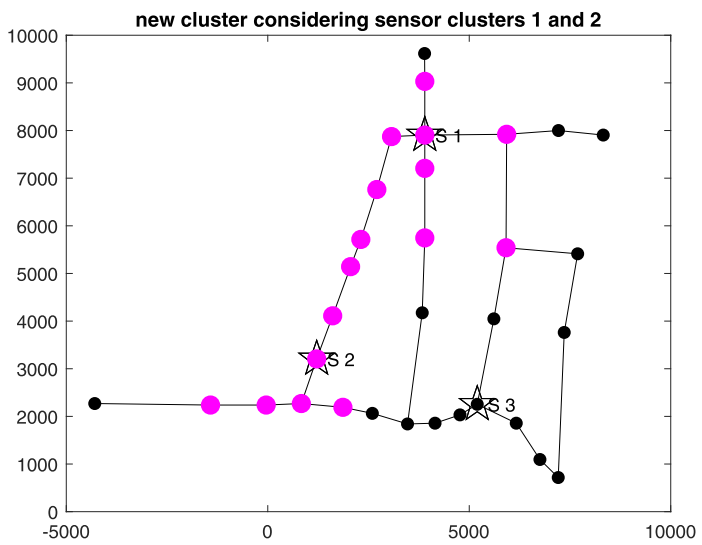

Fig. 3. $\mathcal{K}_{1}^{(\mathcal{W})}$ cluster in the simplified Hanoi WDN between clusters $\mathcal{K}_{1}^{(\mathcal{L})}$ and $\mathcal{K}_{2}^{(\mathcal{L})}$.

\section{Expanded Probability Mass Function for Leak Localization}

Now, we have the three sets of hypotheses $\mathcal{H}, \mathcal{L}$, and $\mathcal{W}$. On the one hand, we have the set of singletons $\mathcal{H}$ where the probability mass functions of each node are computed using (21). On the other hand, we have the sets $\mathcal{L}$ and $\mathcal{W}$ with clustered nodes but without probability mass functions assigned to them yet.

To assign probabilities into each set of composed hypotheses $\mathcal{L}$ and $\mathcal{W}$, the mean of the mass probabilities from the set $\mathcal{H}$ of each node inside the cluster is computed as

$$
m\left(\mathcal{L}_{i}\right)=\frac{\sum_{v_{j}^{(2)} \in \mathcal{K}_{i}^{(\mathcal{L})} m\left(\mathcal{H}_{j}\right)}}{\left|\mathcal{K}_{i}^{(\mathcal{L})}\right|}
$$

and the same is done for the composed hypotheses in $\mathcal{W}$.

Then, the new extended set of mass probabilities $m\left(\mathcal{H}^{\prime}\right)$ from the extended set of hypothesis $\mathcal{H}^{\prime}=\{\mathcal{H}, \mathcal{L}, \mathcal{W}\}$ with size $\left|\mathcal{H}^{\prime}\right|=n_{n}+n_{s}+w$ is normalized according to

$$
m\left(\mathcal{H}_{i}^{\prime}\right)=\frac{m\left(\mathcal{H}_{i}^{\prime}\right)}{\sum_{j=1}^{n_{n}} m\left(\mathcal{H}_{j}\right)+\sum_{j=1}^{n_{s}} m\left(\mathcal{L}_{j}\right)+\sum_{j=1}^{w} m\left(\mathcal{W}_{j}\right)}
$$

in order to fulfill the condition $\sum_{i=1}^{\left|\mathcal{H}^{\prime}\right|} m\left(\mathcal{H}_{i}^{\prime}\right)=1$.

Thus, considering the simplified Hanoi WDN, as $n_{n}=31$, $n_{s}=3$, and the number of additional clusters $w=(3 ! / 2 ! 1 !)=3$, the number of hypotheses of the extended set is $\left|\mathcal{H}^{\prime}\right|=37$.

\section{E. Time Leak Localization Reasoning}

Given a sequence of pmfs of the hypotheses set $\mathcal{H}^{\prime}$ at different time instants $t_{1}, t_{2}, \ldots, t_{N}$, the pmfs of the hypotheses set $\mathcal{H}^{\prime}$ considering all the samples can be computed by Dempster's rule following (19) to perform a time reasoning and obtain as a result an enhanced diagnosis as:

$$
m_{t_{1}, \ldots, t_{N}}\left(\mathcal{H}^{\prime}\right)=m_{t_{1}}\left(\mathcal{H}^{\prime}\right) \oplus m_{t_{2}}\left(\mathcal{H}^{\prime}\right) \oplus \cdots \oplus m_{t_{N}}\left(\mathcal{H}^{\prime}\right)
$$

As an illustrative example, we will describe the computation of probability mass function associated with leak in node 1 of the simplified WDN of Hanoi considering pressure residuals computed from measurements at initial time instant $t_{1}$ and 
time instant $t_{2}$, i.e., $m_{t_{1}, t_{2}}\left(\mathcal{H}_{1}^{\prime}\right)$. As node 1 belongs to the group associated with sensed node $2\left(\mathcal{K}_{1}^{(\mathcal{L})}\right)$ and to additional cluster $\mathcal{K}_{1}^{(\mathcal{W})}$ depicted in Fig. 3 that correspond with hypotheses $\mathcal{H}_{32}^{\prime}$ and $\mathcal{H}_{35}^{\prime}$, the probability mass function of these hypothesis will also be involved in the computation of the pmf of the singleton hypothesis $\mathcal{H}_{1}^{\prime}$. Applying (31), the probability mass function associated with leak in node 1 can be computed as

$$
\begin{aligned}
m_{t_{1}, t_{2}}\left(\mathcal{H}_{1}^{\prime}\right)=\frac{1}{\Lambda} \times & \left(m_{t_{1}}\left(\mathcal{H}_{1}^{\prime}\right) m_{t_{2}}\left(\mathcal{H}_{1}^{\prime}\right)\right. \\
& \left.+m_{t_{1}}\left(\mathcal{H}_{1}^{\prime}\right)\left(m_{t_{2}}\left(\mathcal{H}_{32}^{\prime}\right)\right)+m_{t_{2}}\left(\mathcal{H}_{35}^{\prime}\right)\right) \\
& \left.\left.+m_{t_{2}}\left(\mathcal{H}_{1}^{\prime}\right)\left(m_{t_{1}}\left(\mathcal{H}_{32}^{\prime}\right)\right)+m_{t_{1}}\left(\mathcal{H}_{35}^{\prime}\right)\right)\right) .
\end{aligned}
$$

To select a given node among all the candidates, the resulting hypothesis with larger probability is chosen as

$$
\hat{\jmath}=\underset{j \in\left\{1, \ldots, n_{n}\right\}}{\arg \max }\left\{m_{t_{1}, \ldots, t_{N}}\left(\mathcal{H}_{j}^{\prime}\right)\right\} .
$$

\section{SUMMARY}

The application of the proposed leak localization technique can be divided into offline and online stages.

Note that the leak detection task is not considered in this work, neither the problem of data validation, which are problems that have to be addressed first.

The offline stage can be summarized as follows.

1) Use the topological information to calculate the shortest weighted pipe distance between each pair of nodes (11) to generate the $\overline{\mathcal{D}}$ matrix.

2) Perform the clustering process to identify the clusters $\mathcal{L}$ and $\mathcal{W}$ that will be used as a composed hypothesis.

3) Use historical leak-free data to fit the models (4) that predict the pressure behavior without leak according to the actual network operational conditions $\mathbf{c}$.

The online stage that is triggered when a leak is detected can be summarized as follows.

1) Compute the expected pressure behavior without leaks at the sensed nodes using the actual operating conditions and the fit models (4).

2) Apply the Kriging interpolation technique to generate the expected reference (no-leak) map using the predicted values.

3) Apply the Kriging interpolation technique using the current pressure measurements to generate the current (leak) pressure map.

4) Compute the difference between the two maps (16).

5) Compute the probability mass functions of the singleton hypotheses $\mathcal{H}$ by means of (21) and compute the pmfs of composed hypotheses $\mathcal{L}$ and $\mathcal{W}$ by means of (29).

6) Build the expanded set $\mathcal{H}^{\prime}$ and compute the normalized pmfs of the hypotheses of $\mathcal{H}^{\prime}$ using (30).

7) Apply the DS reasoning (31) to combine diagnosis from different time instants.

8) Select a given node among all the candidate nodes using (33).

The whole procedure is summarized in the flowcharts presented in Figs. 4 and 5.

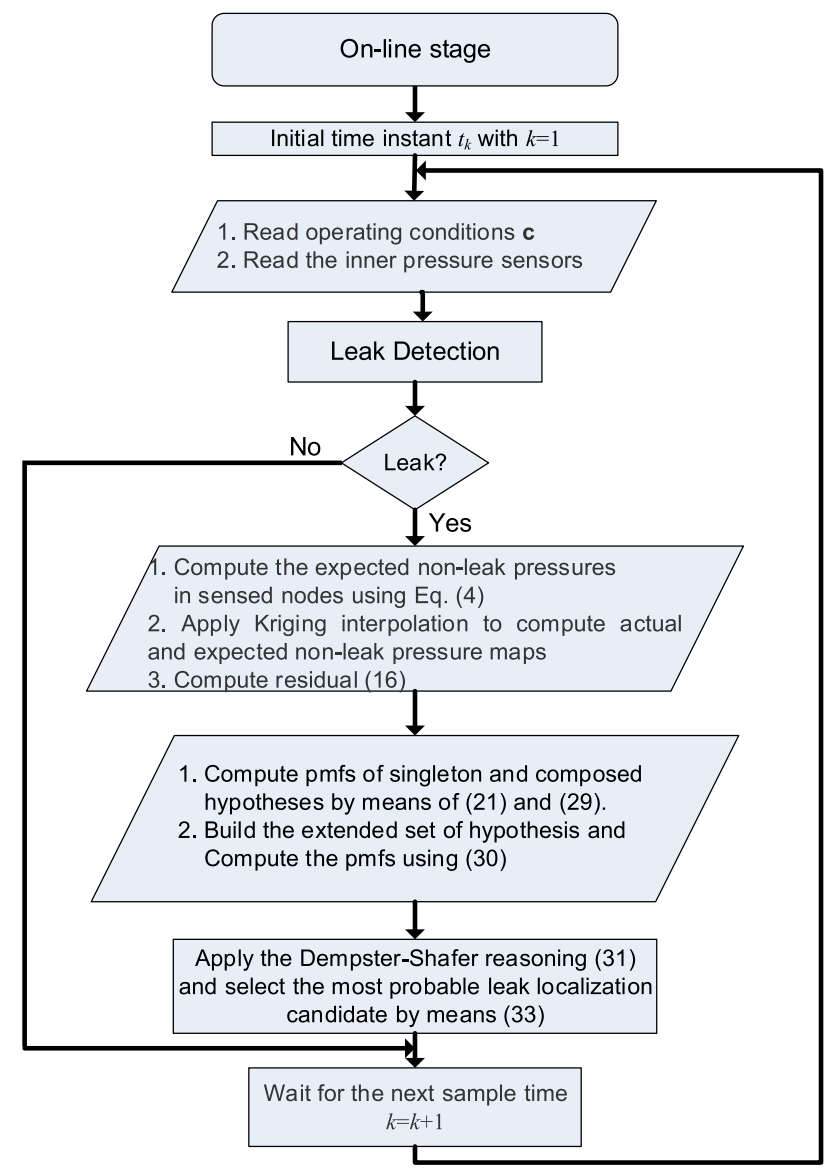

Fig. 4. Proposed method flowchart: online stage.

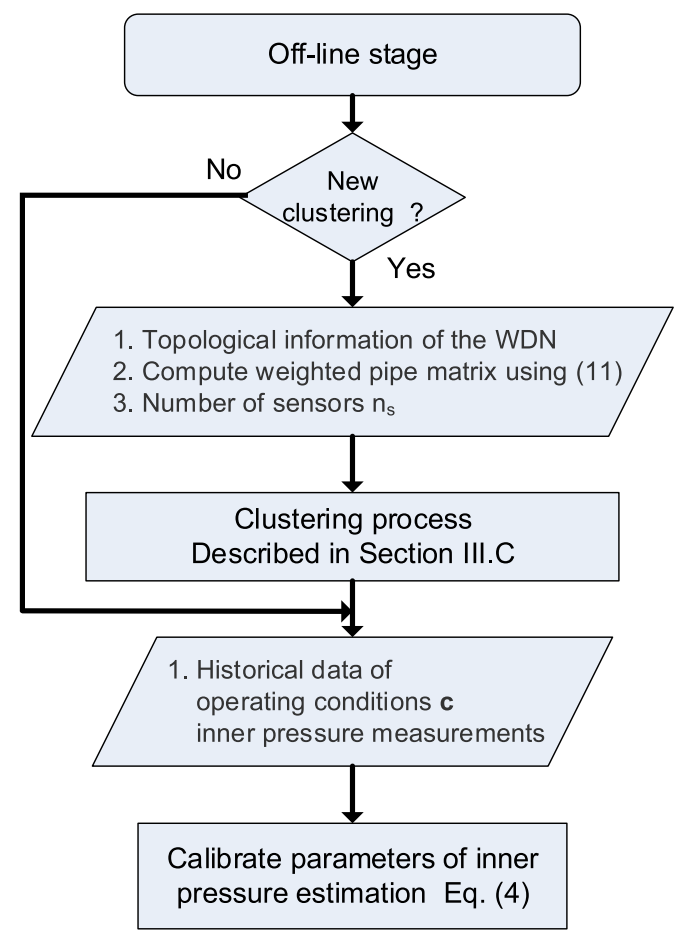

Fig. 5. Proposed method flowchart: offline stage.

\section{CAse Studies}

The proposed leak localization technique is tested in two DMAs from the WDN of the city of Madrid (Spain), where the water company in charge has produced real case studies of 


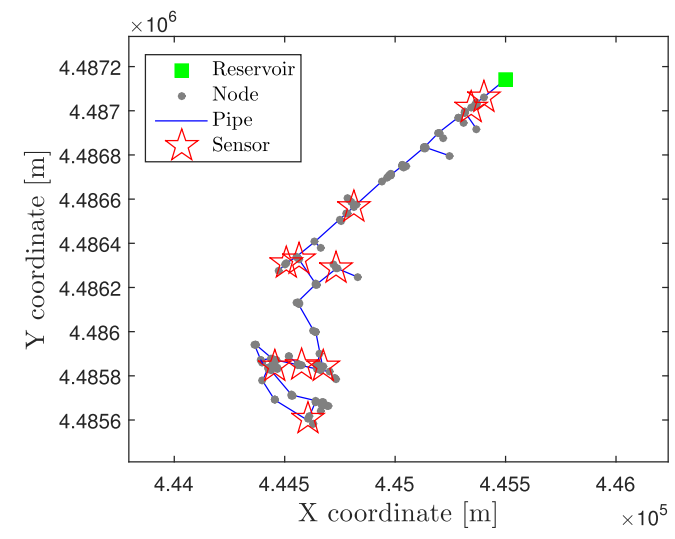

Fig. 6. Madrid DMA1 topological network.

water leaks by using fire hydrants. These DMAs are equipped with flow and pressure sensors at the inlet and the pressure sensors inside the network. Also, a flowmeter and a valve at the leak point are installed to measure and control the magnitude of the leak. For every DMA, the leak magnitude set point was set as a value that the water company considered challenging in leak localization terms. In all the cases, the installed sensors have a sampling rate of two minutes, and they have a resolution of $0.1[\mathrm{mwc}]$ in the case of pressure sensors and a resolution of $0.1[1 / \mathrm{s}]$ in the case of flow sensors. The results of the proposed leak localization method have been compared with the ones provided by other two methods: the proposed method considering the Bayesian reasoning instead of the DS one (i.e., without considering cluster hypothesis) and a well-accepted method proposed in the literature [36] that is based on the use of a hydraulic model to generate leak signatures to be matched with current measurements according to the Angle metric.

\section{A. Madrid DMAI}

Madrid DMA1 is a small network formed by one reservoir that feeds the network with water by elevation, 169 consumer nodes, and 173 pipes. Ten pressure sensors are placed inside at nodes with indexes 146, 145, 148, 162, 153, 155, 156, 157, 158, and 168. The topology and the location of the sensors installed are depicted in Fig. 6. Two periods of data, with and without leak, were recorded. Measurements without leak were recorded starting the December 19, 2016, at 4:00 P.M. to December 22, 2016, at 0:58 A.M., whereas the leak event was recorded from December 22, 2016, at 4:00 A.M. to December 23, 2016, at 8:58 A.M. The controlled leak of set point magnitude is $0.8[1 / \mathrm{s}]$ approximately. The leak and the inlet measurements are depicted in Fig. 7; in addition, a detail of the leak flow is provided in Fig. 8.

The measurements inside the network are depicted in Figs. 9 and 10 for both periods (set 1 comprises the nodes from 146 to 153 and set 2 from 155 to 168 ).

First, the data are downsampled to each hour by computing the average of the value measurements inside. Then, the $57 \mathrm{~h}$ of data without leak are used to fit the models described in (4) for each node equipped with a sensor by means of the least-squares fitting technique, which, in this case, leads to ten
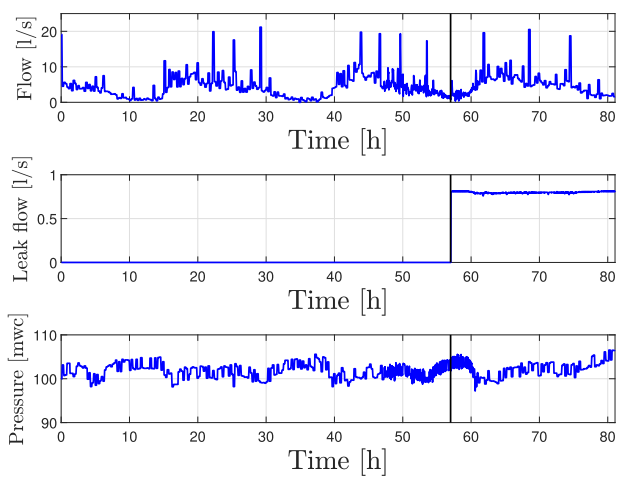

Fig. 7. Madrid DMA1 inlet flow, leak flow, and pressure rate measurements without leak (before black line) and with leak (after black line).

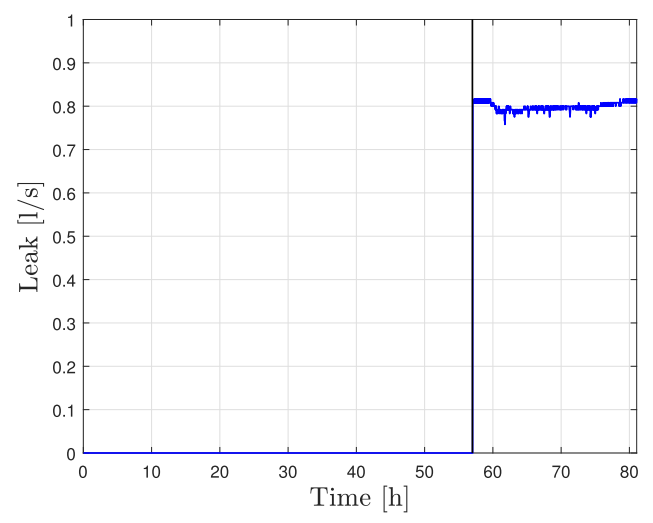

Fig. 8. Madrid DMA1 leak flow detail.

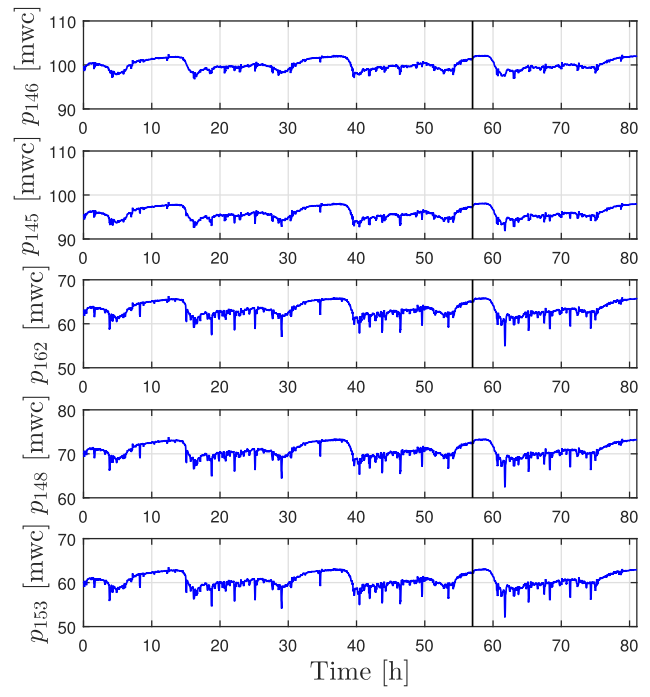

Fig. 9. Madrid DMA1 pressure measurements (set 1) without leak (before black line) and with leak (after black line).

pressure models to predict the internal pressure under no-leak conditions through the inlet operational conditions.

To calculate the shortest weighted pipe distance contained in the $\overline{\mathcal{D}}$ matrix, the underlying network graph $\mathcal{G}$ is used but now considering it as undirected. Then, to fill in the matrix, Dijkstra's algorithm [37] is used to compute the shortest weighted pipe distance between each pair of nodes. 


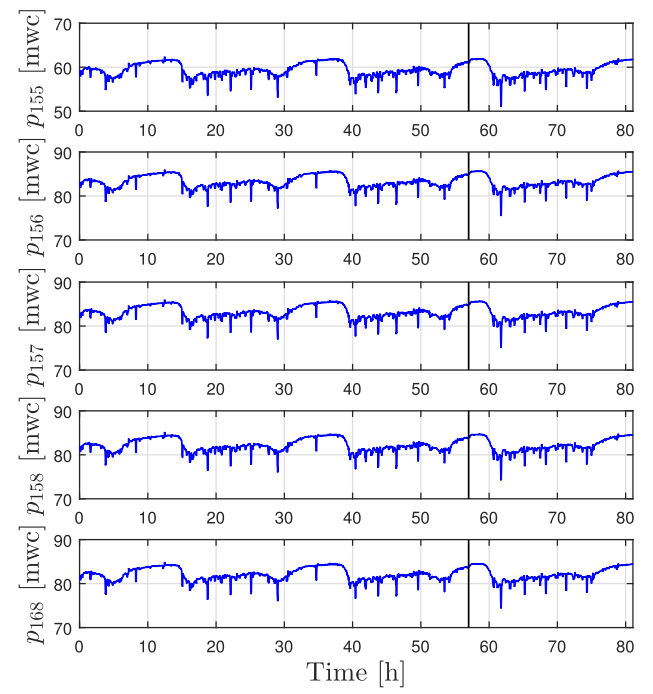

Fig. 10. Madrid DMA1 pressure measurements (set 2) without leak (before black line) and with leak (after black line).

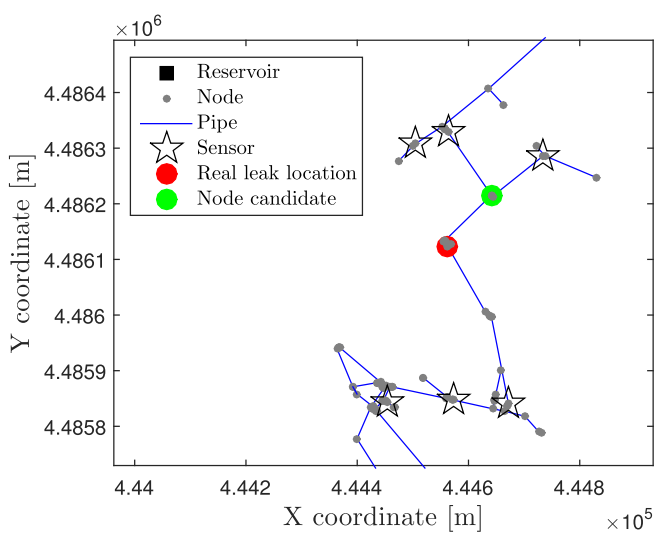

Fig. 11. Leak localization results in Madrid DMA1 real case.

To estimate the pressure in the nodes inside the network that has no pressure sensors installed, the Kriging interpolation technique has been implemented by means of the DACE MATLAB toolbox [23]. The total number of clusters $\mathcal{K}^{(\mathcal{W})}$, $w$, is seven.

The proposed leak localization technique is applied to the Madrid DMA1 real case, where the real leak is placed in the node 165 . At the end of the $28 \mathrm{~h}$ that the leak lasted, the method provides the node 44 as the candidate to be the leaking node. Compared with the leak location, the candidate node presents a geometric linear distance of $120.8 \mathrm{~m}$ and a pipe distance of $132.8 \mathrm{~m}$ to the real leak location. The result is depicted in Fig. 11, where a zoomed-in representation of an area of the network surrounding the real leak location and including the proposed candidate node is presented.

The results obtained by the proposed method (labeled as "DS"), the proposed method without considering cluster hypothesis (labeled as "Bayesian"), and the Angle method [36] (labeled as "Angle") are presented in Table I. In Table I, it can be noted that the proposed method provides better results
TABLE I

MADRID DMA1 LEAK LOCALIZATION RESULTS COMPARISON

\begin{tabular}{l||cc} 
Method & Linear distance [m] & Pipe distance [m] \\
\hline \hline DS & 120.8 & 132.8 \\
Bayesian & 276.2 & 474.1 \\
Angle & 303.3 & 363.4
\end{tabular}

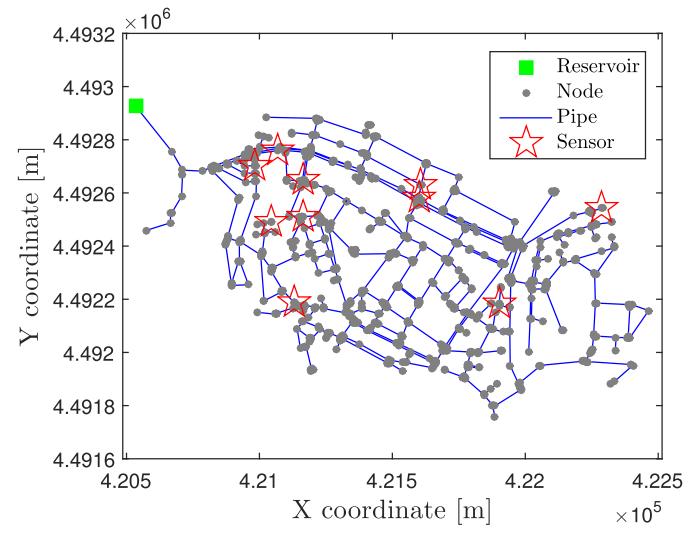

Fig. 12. Madrid DMA2 topological network.
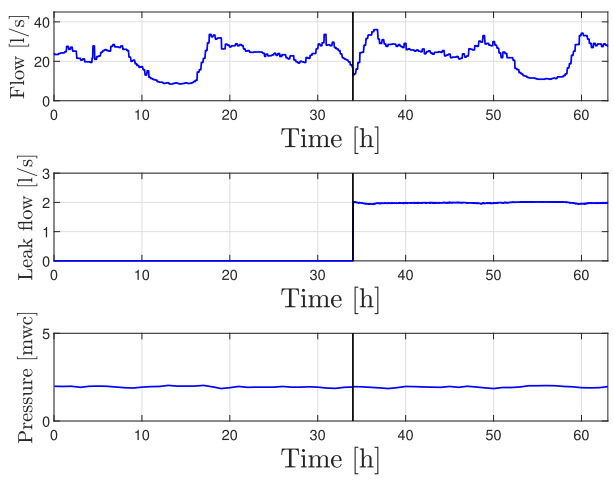

Fig. 13. Madrid DMA2 inlet flow, leak flow, and pressure rate measurements without leak (before black line) and with leak (after black line).

(measured in linear and pipe distance to the leaky node) than the other two methods.

\section{B. Madrid DMA2}

Madrid DMA2 is a medium to large network formed by one reservoir that feeds the network by elevation, 1031 consumer nodes, and 1100 pipes. Ten pressure sensors are placed inside at nodes with indexes 886, 877, 864, 971, 880, 898, 917, 933, 943 , and 948. The topology and the location of the sensors installed are depicted in Fig. 12.

Measurements have been taken starting from January 30, 2017 , at 2:00 A.M. to January 31,2017 , at 11:28 P.M. under no-leak conditions, and data with a controlled leak of set point magnitude of $2[1 / \mathrm{s}]$ were taken from February 1, 2017, at 6:00 P.M. to February 2, 2017, at 11:58 A.M. The inlet measurements and the leak flow rate are depicted in Fig. 13; in addition, a detail of the leak flow is provided in Fig.14.

The pressure measurements inside the network are depicted in Figs. 15 and 16. 


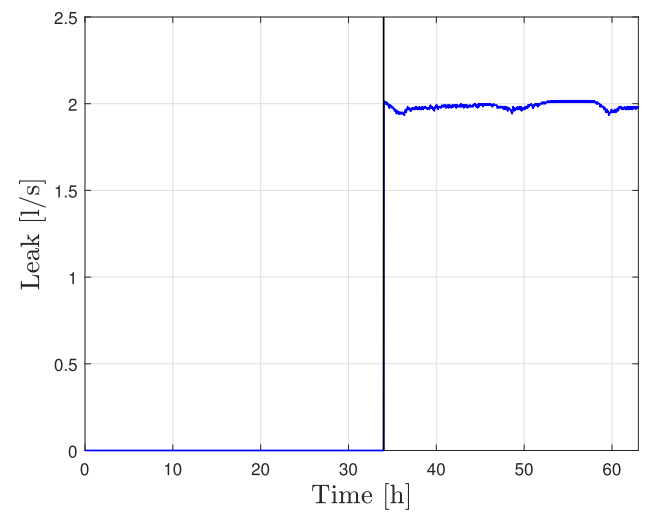

Fig. 14. Madrid DMA2 leak flow detail.

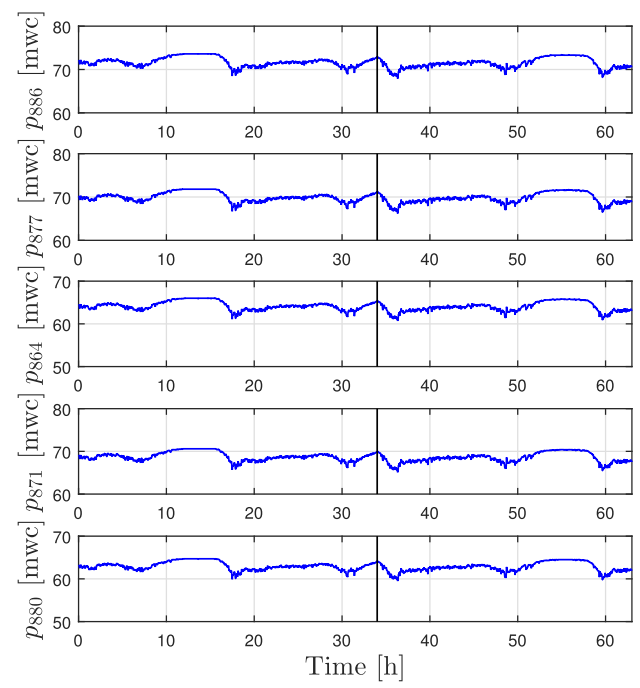

Fig. 15. Madrid DMA2 pressure measurements (set 1) without leak (before black line) and with leak (after black line).

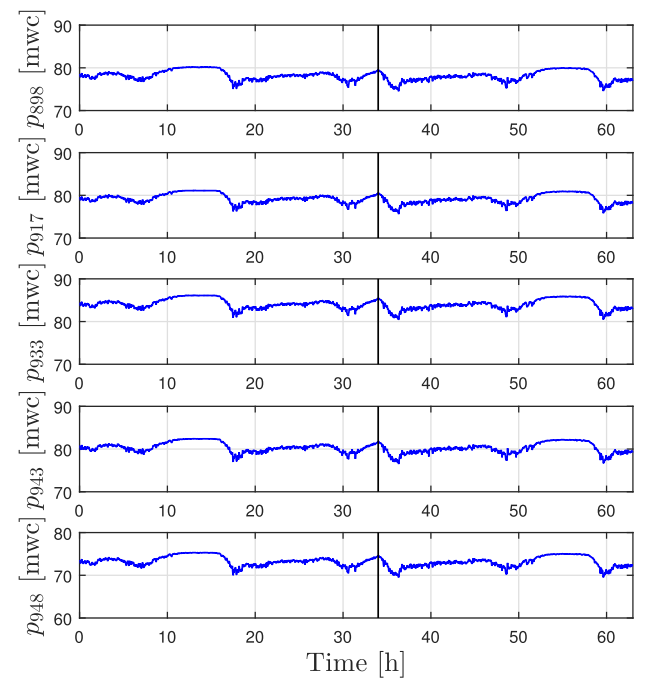

Fig. 16. Madrid DMA2 pressure measurements (set 2) without leak (before black line) and with leak (after black line).

As in the previous case study, the hourly average is computed, and the first $54 \mathrm{~h}$ are used to fit the ten pressure models. In this case, 16 clusters $\mathcal{K}^{(\mathcal{W})}$ have been generated. The diagnosis through $18 \mathrm{~h}$ of leaky data points to the node 5 as a candidate, whereas the leak is actually in node 882 .

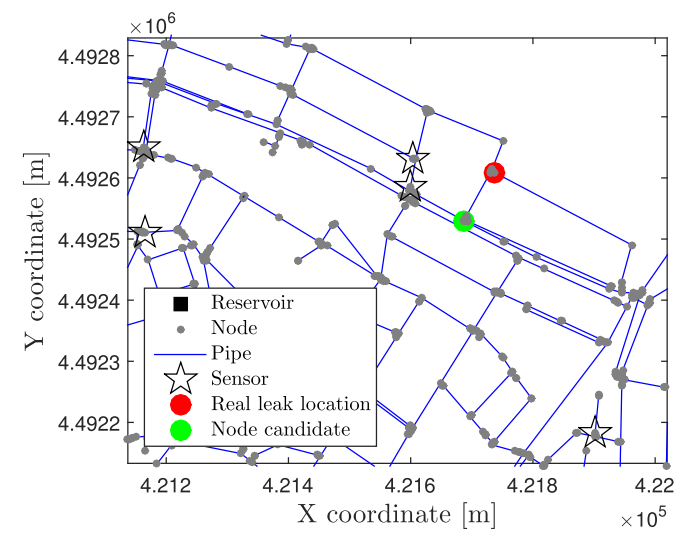

Fig. 17. Leak localization results in Madrid DMA2 real case.

TABLE II

MADRID DMA2 LEAK LOCALIZATION RESULTS COMPARISON

\begin{tabular}{l||cc} 
Method & Linear distance $[\mathrm{m}]$ & Pipe distance $[\mathrm{m}]$ \\
\hline \hline DS & 93.4 & 98.8 \\
Bayesian & 506.9 & 758.8 \\
Angle & 594.6 & 679.2
\end{tabular}

In this case, the geometric linear distance is $93.4 \mathrm{~m}$, while the pipe distance is $98.8 \mathrm{~m}$ to the real leak location. The result is depicted in Fig. 17.

The results obtained by the proposed method and the other two methods are presented in Table II. In Table II, it can be noted that the proposed method provides better results (measured in linear and pipe distance to the leaky node) than the other two methods.

\section{Results and Discussion}

From the leak localization results obtained in the two real DMAs of Madrid summarized in Tables I and II, we can state that the proposed method outperforms the Angle method presented in [36]. The proposed method is based on the fact that when a leak occurs, and there is a loss of pressure across the network, especially in the places where the additional flow produced by the leak passes through the network until it arrives at the leak location. This loss of pressure is observed by means of the differences (residuals) between the estimated pressures (considering nonleak scenario) and actual pressures measured in inner sensors. The pressures of nonmeasured inner nodes are estimated by the Kriging method; therefore, there is an additional error in the computation of the residuals of nonmeasured nodes that increases with the distance of these nodes from sensed nodes. This additional error can produce a deviation in the leak localization to nodes that are closer to sensed nodes. The additional information of clusters by means of the DS reasoning improves significantly the accuracy in the leak localization. Finally, in absolute terms, the accuracy obtained by the proposed method (around $100[\mathrm{~m}]$ of error in the leak localization) is considered reasonable for the water companies because it is possible to send operators to search and exactly pinpoint the leak through acoustic sensors in this 
bounded area of the DMA since this technique is too time consuming to be applied across all the DMA.

\section{CONCLUSION}

In this article, a new methodology for a data-driven leak localization problem in WDNs has been presented and tested. The proposed approach is based on the hydraulic characteristics that appear when a leak in a particular place of the network occurs. A data-driven model has been used for the prediction of internal pressures without leak based on past measurements. The Kriging interpolation has been proposed to overcome the problem of limited sensor measurements in the network. The application of the DS reasoning is proposed to enhance the leak localization performance by taking into account the time evolution of the residuals. The method has been successfully tested in two DMAs of real water network providing better leak localization results compared with two other alternative methods. One of them considers the same approach but using time reasoning based on the Bayes rule, while the other is based on the use of a hydraulic model to generate leak signatures to be matched with current measurements according to the Angle metric. Both methods are outperformed by the proposed approach.

The performance of the proposed methodology has been successfully tested with real measurement data from three DMAs of the Madrid WDN.

As future work, the proposed leak localization method will be tested in other DMAs with different topologies and sizes. In addition, it will be interesting to investigate the application of the proposed data-driven approach to monitoring water pollution. Also, the case of multiple leaks occurring at the same time will be considered.

\section{REFERENCES}

[1] R. Puust, Z. Kapelan, D. A. Savic, and T. Koppel, "A review of methods for leakage management in pipe networks," Urban Water J., vol. 7, no. 1, pp. 25-45, Feb. 2010.

[2] D. G. Eliades and M. M. Polycarpou, "A fault diagnosis and security framework for water systems," IEEE Trans. Control Syst. Technol., vol. 18 , no. 6 , pp. $1254-1265$, Nov. 2010.

[3] Z. Y. Wu and P. Sage, "Water loss detection via genetic algorithm optimization-based model calibration," in Proc. Water Distribution Syst. Anal. Symp. Reston, VA, USA: ASCE, 2006, pp. 1-11.

[4] J. Yang, Y. Wen, and P. Li, "Leak location using blind system identification in water distribution pipelines," J. Sound Vib., vol. 310, nos. 1-2, pp. 134-148, Feb. 2008.

[5] H. V. Fuchs and R. Riehle, "Ten years of experience with leak detection by acoustic signal analysis," Appl. Acoust., vol. 33, no. 1, pp. 1-19, 1991.

[6] J. M. Muggleton, M. J. Brennan, and R. J. Pinnington, "Wavenumber prediction of waves in buried pipes for water leak detection," J. Sound Vib., vol. 249, no. 5, pp. 939-954, Jan. 2002.

[7] J. Mashford, D. D. Silva, D. Marney, and S. Burn, "An approach to leak detection in pipe networks using analysis of monitored pressure values by support vector machine," in Proc. 3rd Int. Conf. Netw. Syst. Secur., 2009, pp. 534-539.

[8] A. Soldevila, J. Blesa, S. Tornil-Sin, E. Duviella, R. M. Fernandez-Canti, and V. Puig, "Leak localization in water distribution networks using a mixed model-based/data-driven approach," Control Eng. Pract., vol. 55, pp. 162-173, Oct. 2016.

[9] A. Soldevila, R. M. Fernandez-Canti, J. Blesa, S. Tornil-Sin, and V. Puig, "Leak localization in water distribution networks using Bayesian classifiers," J. Process Control, vol. 55, pp. 1-9, Jul. 2017.
[10] D. Wachla, P. Przystalka, and W. Moczulski, "A method of leakage location in water distribution networks using artificial neuro-fuzzy system," IFAC-PapersOnLine, vol. 48, no. 21, pp. 1216-1223, 2015.

[11] R. Pérez, V. Puig, J. Pascual, J. Quevedo, E. Landeros, and A. Peralta, "Methodology for leakage isolation using pressure sensitivity analysis in water distribution networks," Control Eng. Pract., vol. 19, no. 10, pp. 1157-1167, Oct. 2011.

[12] P. Cugueró-Escofet, J. Blesa, R. Pérez, M. A. Cugueró-Escofet, and G. Sanz, "Assessment of a leak localization algorithm in water networks under demand uncertainty," IFAC-PapersOnLine, vol. 48, no. 21, pp. 226-231, 2015.

[13] J. Blesa and R. Pérez, "Modelling uncertainty for leak localization in water networks," IFAC-PapersOnLine, vol. 51, no. 24, pp. 730-735, 2018.

[14] M. V. Casillas, L. E. G. Castañón, and V. P. Cayuela, "Extended-horizon analysis of pressure sensitivities for leak detection in water distribution networks," in Proc. 8th IFAC Symp. Fault Detection, Supervision Saf. Tech. Processes. Amsterdam, The Netherlands: Elsevier, 2012, pp. $570-575$.

[15] J. Gross, J. L. Yellen, and M. Anderson, Graph Theory and Its Applications. New York, NY, USA: CRC Press, 2005.

[16] P. K. Swamee and A. K. Sharma, Design of Water Supply Pipe Networks, 1st ed. Hoboken, NJ, USA: Wiley, 2008.

[17] T. N. Jense, C. S. Kallesoe, J. D. Bendtse, and R. Wisniewsk, "Plug-andplay commissionable models for water networks with multiple inlets," in Proc. Eur. Control Conf. (ECC), Jun. 2018, pp. 1-6.

[18] T. N. Jensen, C. S. Kallesoe, J. D. Bendtsen, and R. Wisniewski, "Iterative learning pressure control in water distribution networks," in Proc. IEEE Conf. Control Technol. Appl. (CCTA), Copenhagen, Denmark, Aug. 2018, pp. 583-588.

[19] L. Ljung, System Identification-Theory for the User, 2nd ed. Upper Saddle River, NJ, USA: Prentice-Hall, 1999.

[20] J. P. C. Kleijnen, "Regression and kriging metamodels with their experimental designs in simulation: A review," Eur. J. Oper. Res., vol. 256, no. 1, pp. 1-16, Jan. 2017.

[21] A. Soldevila, J. Blesa, R. M. Fernandez-Canti, S. Tornil-Sin, and V. Puig, "Data-driven approach for leak localization in water distribution networks using pressure sensors and spatial interpolation," Water, vol. 11, no. 7 , p. 1500,2019 . [Online]. Available: https://www.mdpi.com/20734441/11/7/1500

[22] C. S. Kallesoe, T. N. Jensen, and R. Wisniewski, "Adaptive reference control for pressure management in water networks," in Proc. Eur. Control Conf. (ECC), Jul. 2015, pp. 3268-3273.

[23] H. B. Nielsen, S. N. Lophaven, and J. Søndergaard, "DACE-A MATLAB kriging toolbox," in Richard Petersens Plads, Building, vol. 321. Lyngby, Denmark: Informatics and Mathematical Modelling, Technical University of Denmark, DTU, 2002. [Online]. Available: http://www2.imm.dtu.dk/pubdb/p.php?1460

[24] M. V. Casillas, L. E. Garza-Castanon, and V. Puig, "Extended-horizon analysis of pressure sensitivities for leak detection in water distribution networks: Application to the barcelona network," in Proc. Eur. Control Conf. (ECC), Zürich, Switzerland, no. 1, Jul. 2013, pp. 401-409.

[25] T. N. Jensen and C. S. Kallesoe, "Application of a novel leakage detection framework for municipal water supply on AAU water supply lab," in Proc. 3rd Conf. Control Fault-Tolerant Syst. (SysTol), Sep. 2016, pp. $428-433$.

[26] M. Romano, K. Woodward, and Z. Kapelan, "Statistical process control based system for approximate location of pipe bursts and leaks in water distribution systems," Procedia Eng., vol. 186, pp. 236-243, 2017.

[27] C. S. Kallesoe and T. N. Jensen, "On the relation between leakage location and network pressures," in Proc. IEEE Conf. Control Technol. Appl. (CCTA), Aug. 2018, pp. 571-576.

[28] G. Shafer, A Mathematical Theory of Evidence, vol. 42. Princeton, NJ, USA: Princeton Univ. Press, 1976.

[29] G. Shafer, "Dempster's rule of combination," Int. J. Approx. Reasoning, vol. 79, pp. 26-40, Dec. 2016.

[30] R. Gonzalez, F. Qi, and B. Huang, Process Control System Fault Diagnosis: A Bayesian Approach. Chichester, U.K.: Wiley, 2016.

[31] J. Bicik, Z. Kapelan, C. Makropoulos, and D. A. Savić, "Pipe burst diagnostics using evidence theory," J. Hydroinformatics, vol. 13, no. 4, pp. 596-608, Oct. 2011.

[32] R. Sadiq and M. J. Rodriguez, "Interpreting drinking water quality in the distribution system using Dempster-Shafer theory of evidence," Chemosphere, vol. 59, no. 2, pp. 177-188, Apr. 2005. 
[33] O. Fujiwara and D. B. Khang, "A two-phase decomposition method for optimal design of looped water distribution networks," Water Resour. Res., vol. 26, no. 4, pp. 539-549, Apr. 1990, doi: 10.1029/WR026i004p00539.

[34] A. Dempster, "A generalization of Bayesian inference," in Classic Works of the Dempster-Shafer Theory of Belief Functions. Berlin, Germany: Springer, 2008, pp. 73-104.

[35] J. Blesa, F. Nejjari, and R. Sarrate, "Robust sensor placement for leak location: Analysis and design," J. Hydroinform., vol. 18, no. 1, pp. 136-148, Jan. 2016.

[36] M. V. Casillas Ponce, L. E. Garza Castañón, and V. P. Cayuela, "Model-based leak detection and location in water distribution networks considering an extended-horizon analysis of pressure sensitivities," J. Hydroinform., vol. 16, no. 3, pp. 649-670, 2013.

[37] E. W. Dijkstra, "A note on two problems in connexion with graphs," Numerische Math., vol. 1, no. 1, pp. 269-271, Dec. 1959.

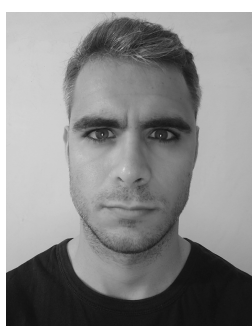

Adrià Soldevila received the bachelor's degree in industrial electronics engineering, the master's degree in automatics and electronics engineering, and the Ph.D. degree in control, vision, and robotics from the Universitat Politècnica de Catalunya (UPC), Barcelona, Spain, in 2012, 2014, and 2018, respectively.

He is currently the Chief Technical Officer of FACTIC, Inc., Barcelona. His research interest involves the fault diagnosis of dynamic systems by means of machine learning techniques.

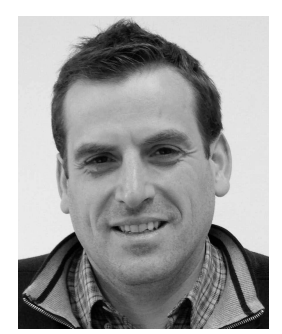

Joaquim Blesa received the Telecommunications Engineering degree and the Ph.D. degree in control, vision, and robotics from the Universitat Politècnica de Catalunya (UPC), Barcelona, Spain, in 1997 and 2011, respectively.

He is currently a Serra Húnter Fellow with the Automatic Control Department, UPC. He has participated in several national and European projects and has published several articles in international conference proceedings and scientific journals. His current research interests include robust identification in the automatic control field and the fault diagnosis of complex systems.

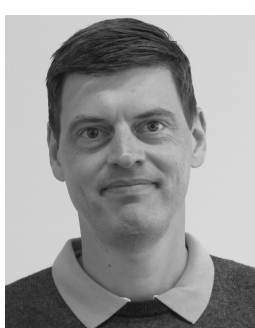

Tom Nørgaard Jensen received the M.Sc. and $\mathrm{Ph} . \mathrm{D}$. degrees in automatic control from Aalborg University, Aalborg, Denmark, in 2008 and 2012, respectively.

$\mathrm{He}$ is currently an Automation Engineer with Alfa Laval, Aalborg. He has been working with the research and development of the area of control and estimation in hydraulic network applications, such as water supply systems and district heating systems. His research interests include control of nonlinear systems, estimation, and fault detection in networked systems.

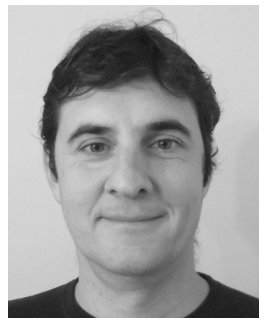

Sebastian Tornil-Sin received the Computer Engineering degree and the Ph.D. degree in control, vision, and robotics from the Universitat Politècnica de Catalunya (UPC), Barcelona, Spain, in 1996 and 2006, respectively.

$\mathrm{He}$ is currently an Associate Professor with the Automatic Control Department (ESAII), UPC where he is also a member of the Advanced Control Systems Research Group. His main research interests include the application of interval analysis in the automatic control field and the fault diagnosis and fault-tolerant control of dynamic systems.

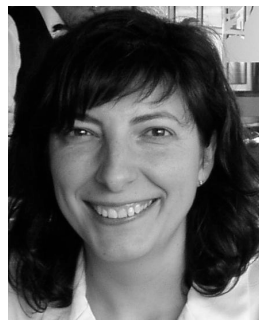

Rosa M. Fernández-Cantí received the Telecommunications Engineering degree and the Ph.D. degree in control engineering from the Technical University of Catalonia (UPC), Barcelona, Spain, in 1994 and 2013, respectively.

She is currently an Associate Professor with the Department of Automatic Control (ESAII), UPC. She is also a member of the Intelligent Control Systems/Advanced Control Systems (SIC/SAC) Research Group, UPC, Terrassa, Spain. Her main research interests include the application of the Bayesian tools to the identification and fault diagnosis of dynamic systems.

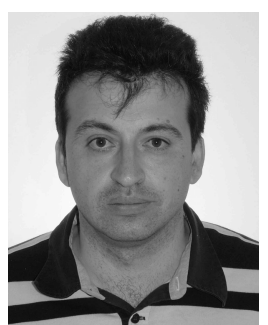

Vicenç Puig received the B.Sc./M.Sc. degree in telecommunications engineering and the $\mathrm{Ph} . \mathrm{D}$. degree in automatic control, vision, and robotics from the Universitat Politècnica de Catalunya (BarcelonaTech) (UPC), Barcelona, Spain, in 1993 and 1999, respectively.

He is currently a Full Professor with the Automatic Control Department, UPC, and a Researcher with the Institut de Robòtica i Informàtica Industrial (IRI), CSIC-UPC. He is also the Director of the Automatic Control Department and the Head of the Research Group on Advanced Control Systems (SAC), UPC. He has developed important scientific contributions in the areas of fault diagnosis and fault-tolerant control, using interval and linear-parameter-varying models using set-based approaches. He has participated in more than 20 European and national research projects in the last decade. He has also led many private contracts with several companies and has published more than 140 journal articles as well as over 400 contributions in international conference/workshop proceedings. He has supervised over $20 \mathrm{Ph} . \mathrm{D}$. dissertations and over 50 master's theses/final projects.

Dr. Puig was the General Chair of the third IEEE Conference on Control and Fault-Tolerant Systems (SysTol 2016) and the IPC Chair of IFAC Safeprocess 2018. He is also the Chair of the IFAC Safeprocess TC Committee 6.4. 